\title{
Statistics of turbulent fluctuations in counter-rotating Taylor-Couette flows
}

\author{
Sander G. Huisman, ${ }^{*}$ Detlef Lohse, ${ }^{\dagger}$ and Chao Sun $^{\ddagger}$ \\ Department of Applied Physics and J. M. Burgers Centre for Fluid Dynamics, University of Twente, \\ P. O. Box 217, 7500 AE Enschede, The Netherlands
}

(Received 16 August 2013; published 2 December 2013)

\begin{abstract}
The statistics of velocity fluctuations of turbulent Taylor-Couette flow are examined. The rotation rates of the inner and outer cylinders are varied while keeping the Taylor number fixed to $1.49 \times 10^{12}\left[O(\mathrm{Re})=10^{6}\right]$. The azimuthal velocity component of the flow is measured using laser Doppler anemometry. For each experiment $5 \times 10^{6}$ data points are acquired and carefully analyzed. Using extended self-similarity [Benzi et al., Phys. Rev. E 48, R29 (1993)] the longitudinal structure function exponents are extracted and are found to weakly depend on the ratio of the rotation rates. For the case where only the inner cylinder rotates the results are in good agreement with results measured by Lewis and Swinney [Phys. Rev. E 59, 5457 (1999)] using hot-film anemometry. The power spectra show clear $-5 / 3$ scaling for the intermediate angular velocity ratios $-\omega_{o} / \omega_{i} \in\{0.6,0.8,1.0\}$, roughly $-5 / 3$ scaling for $-\omega_{o} / \omega_{i} \in\{0.2,0.3,0.4,2.0\}$, and no clear scaling law can be found for $-\omega_{0} / \omega_{i}=0$ (inner cylinder rotation only); the local scaling exponent of the spectra has a strong frequency dependence. We relate these observations to the shape of the probability density function of the azimuthal velocity and the presence of a neutral line.
\end{abstract}

DOI: 10.1103/PhysRevE.88.063001

PACS number(s): 47.27.-i, 47.32.Ef

Taylor-Couette (TC) flow, among others like RayleighBénard convection, and von Kármán, pipe, channel, and plate flows, played a pivotal role in exploring fundamental concepts in fluid mechanics [1]. In a TC apparatus, fluid is confined between two independently rotating coaxial cylinders; see Fig. 1. The TC geometry is best described with cylindrical coordinates: radial distance $\rho$, azimuth $\theta$, and height $z$. The driving of the TC apparatus is given by two Reynolds numbers:

$$
\operatorname{Re}_{i, o}=\frac{\omega_{i, o} \rho_{i, o}\left(\rho_{o}-\rho_{i}\right)}{v},
$$

where $\omega$ is the angular velocity defined as $u_{\theta} / \rho, \rho$ the radius, $v$ the kinematic viscosity, and the $i$ and $o$ subscripts denote quantities related to the inner and outer cylinder, respectively. Another way of describing the flow is by a Taylor number $\mathrm{Ta}=\frac{1}{4} \sigma\left(\rho_{o}-\rho_{i}\right)^{2}\left(\rho_{o}+\rho_{i}\right)^{2}\left(\omega_{i}-\omega_{o}\right)^{2} / \nu^{2}$, which is the ratio of centrifugal forces to viscous forces, along with a parameter describing the ratio of the driving velocities, for which we have chosen

$$
a=-\frac{\omega_{o}}{\omega_{i}} .
$$

$\sigma$ is defined as $[(1+\eta) / \sqrt{4 \eta}]^{4}$ with the radius ratio $\eta=\rho_{i} / \rho_{o}$. By measuring the torque $\mathcal{T}$ [3-10] required to maintain constant angular velocity of both cylinders, we can find the power input $(P)$ of our system using $P=\mathcal{T}\left|\omega_{i}-\omega_{o}\right|$. Note that we can measure the torque on either cylinder as it has the same magnitude on the inner and the outer cylinders [11]. As all the energy that enters the system globally will be dissipated by viscous dissipation, the torque can be related to the average energy dissipation rate:

$$
\epsilon=\frac{\text { (power input) }}{\text { (mass) }}=\frac{\mathcal{T}\left|\omega_{i}-\omega_{o}\right|}{\rho_{\text {fluid }} \pi\left(\rho_{o}^{2}-\rho_{i}^{2}\right) L},
$$

\footnotetext{
*s.g.huisman@gmail.com

†d.lohse@utwente.nl

${ }^{\ddagger}$ c.sun@utwente.nl
}

where $\rho_{\text {fluid }}$ is the density of the working fluid and $L$ the length of the cylinders. Using the energy dissipation rate and the viscosity we can now find the average Kolmogorov length scale $[12,13]$ in our flow: $\eta_{K}=\left(v^{3} / \epsilon\right)^{1 / 4}$.

Using hot-film anemometry, Lewis and Swinney [3,14] measured the statistics of velocity fluctuations for the case of inner cylinder rotation for Re up to $5.4 \times 10^{5}$. They found that the energy spectrum does not show power law scaling, and that the structure function exponents-calculated using extended self-similarity [15] — are close to those found in other flows [3]. In this paper we quantify the turbulent statistics of Taylor-Couette flow with various rotation ratios [Eq. (1)] for fixed Taylor number of Ta $=1.49 \times 10^{12}\left[O(\mathrm{Re})=10^{6}\right]$. We used the Twente Turbulent Taylor-Couette facility $\left(\mathrm{T}^{3} \mathrm{C}\right)$ [2], which was filled with water and actively cooled to keep the temperature constant. The $\mathrm{T}^{3} \mathrm{C}$ has an inner cylinder with an outer radius of $\rho_{i}=200 \mathrm{~mm}$ and a transparent outer cylinder with inner radius $\rho_{o}=279 \mathrm{~mm}$, giving a radius ratio of $\eta=0.716$. The cylinders have a height of $L=927 \mathrm{~mm}$, resulting in an aspect ratio of $\Gamma=L /\left(\rho_{o}-\rho_{i}\right)=11.7$. We measured the azimuthal velocity using laser Doppler anemometry (LDA). The advantage of this technique is that it allows for a nonintrusive measurement of a velocity component. For the case of counter-rotation the mean flow direction is not always in a single direction, and using, e.g., a hot-film probe or a pitot tube to measure the local velocity would result in measuring the speed in the wake of the probe. The laser beams go through the outer cylinder and are focused in the middle of the gap $\left(2 \rho=\rho_{i}+\rho_{o}\right)$ at mid-height $(z=L / 2)$, and lie in the $\theta-r$ plane; see also Fig. 1. The water is seeded with tracer particles [16] with a mean radius of $2.5 \mu \mathrm{m}$ and density of $1.03 \mathrm{~g} / \mathrm{cm}^{3}$. This radius is roughly six times smaller than our Kolmogorov length scale. We equate the drag force $F_{\text {drag }}=6 \pi \mu r_{\text {seed }} \Delta u_{\theta}$ and the centrifugal force $F_{\text {cent }}=\left(\rho_{\text {seed }}-\rho_{\text {fluid }}\right)\left(4 \pi r^{3} / 3\right) u_{\theta}^{2} / \rho$ of the seeding particles, and compute $\Delta u_{\theta}=\mid u_{\theta \text {,seed }}-u_{\theta}$,fluid $\mid$ to make sure the particles faithfully follow the flow. For our measurements we find that $\Delta u_{\theta} \approx 40 \mu \mathrm{m} / \mathrm{s}$, which is much 


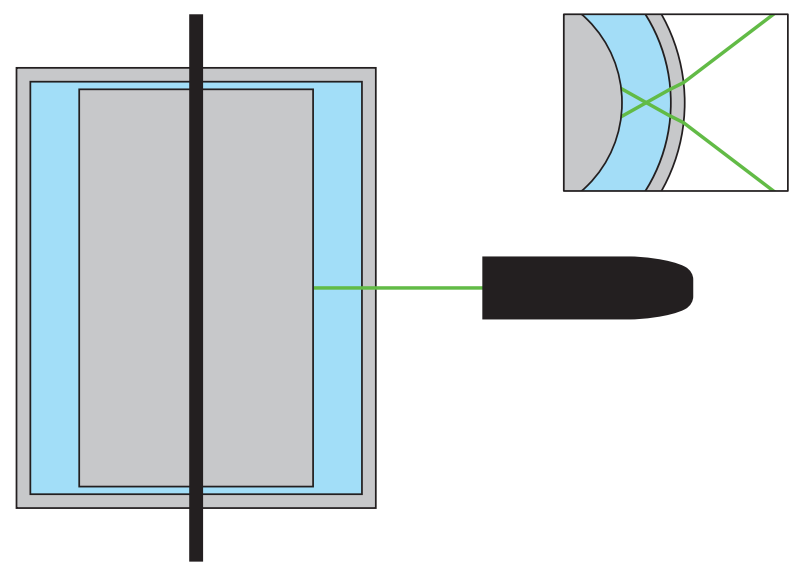

FIG. 1. (Color online) Sketch of the vertical cross section of the Twente Turbulent Taylor-Couette facility $\left(\mathrm{T}^{3} \mathrm{C}\right)$ [2]. The beams of the laser Doppler anemometer (LDA) are in the horizontal plane at middle height, $z=L / 2$. Top right inset: Horizontal cross section showing the beams of the LDA. The beams refract twice on the outer cylinder and intersect at the middle of the gap. The angle between the beams is exaggerated to highlight the refraction on the inner and outer surfaces of the outer cylinder.

smaller than the driving velocities $O\left(\omega_{i} \rho_{i}-\omega_{o} \rho_{o}\right)=10 \mathrm{~m} / \mathrm{s}$, so we are sure that the particles follow the flow. In addition one could calculate the Stokes number; we find that $\mathrm{St}=\tau_{p} / \tau_{\eta}=$ $0.006 \ll 1$ in the worst case $(a=0.3)$. Due to the curvature of the outer cylinder we have to correct the measured velocity by multiplying it with a constant factor. We find this constant numerically by ray-tracing [17] the LDA beams in our optical geometry.

In our experiments we fixed $\Delta \omega \equiv \omega_{i}-\omega_{o}$ (see Table I); the consequence of this is that our Taylor number is fixed, while our Reynolds number varies slightly. For each experiments we acquire $5 \times 10^{6}$ data points. Because the arrival times of LDA measurements are of stochastic nature the time series are then linearly interpolated using twice the average acquisition frequency $\left(2 f_{\mathrm{a}}\right)$, such as to create a time series with equal

TABLE I. Experimental parameters for the various rotation ratios. For each experiment the Taylor number is fixed to $1.49 \times 10^{12}$ and the number of data points is fixed to $5 \times 10^{6} . \omega_{i}$ and $\omega_{o}$ are measured using high-precision magnetic encoders. All the measurements are done at mid-height and in the middle of the gap. $\langle\cdot\rangle_{t}$ denotes averaging over time. The Taylor-Reynolds number is found by combining local velocity and global torque measurements: $\operatorname{Re}_{\lambda}=$ $\sqrt{15 \sigma^{4} / \epsilon \nu}$, where $\epsilon$ comes from the global torque; see Eq. (2). The standard deviation of $u_{\theta}$ is given by $\sigma$ and the kurtosis by $\mu_{4} / \sigma^{4}$.

\begin{tabular}{lrrrrrrrr}
\hline \hline$a$ & $\begin{array}{r}\omega_{i} / 2 \pi \\
(1 / \mathrm{s})\end{array}$ & $\begin{array}{r}\omega_{o} / 2 \pi \\
(1 / \mathrm{s})\end{array}$ & $\begin{array}{c}\operatorname{Re} \\
\left(10^{6}\right)\end{array}$ & $\begin{array}{c}\left\langle u_{\theta}\right\rangle_{t} \\
(\mathrm{~m} / \mathrm{s})\end{array}$ & $\begin{array}{c}\sigma \\
(\mathrm{m} / \mathrm{s})\end{array}$ & $\begin{array}{c}\epsilon \\
\left(\mathrm{m}^{2} / \mathrm{s}^{3}\right)\end{array}$ & $\operatorname{Re}_{\lambda}$ & $\frac{\mu_{4}}{\sigma^{4}}$ \\
\hline 0.0 & 9.99 & 0.00 & 1.38 & 5.08 & 0.31 & 12.9 & 106 & 3.37 \\
0.2 & 8.32 & -1.66 & 1.32 & 2.42 & 0.38 & 15.3 & 144 & 3.00 \\
0.3 & 7.67 & -2.30 & 1.29 & 1.62 & 0.32 & 16.3 & 97 & 3.96 \\
0.4 & 7.13 & -2.85 & 1.27 & 1.48 & 0.35 & 15.8 & 120 & 2.77 \\
0.6 & 6.24 & -3.74 & 1.24 & 0.36 & 0.47 & 13.2 & 240 & 3.02 \\
0.8 & 5.54 & -4.44 & 1.21 & -0.43 & 0.49 & 11.0 & 278 & 2.89 \\
1.0 & 4.99 & -5.00 & 1.19 & -0.83 & 0.43 & 9.2 & 235 & 3.27 \\
2.0 & 3.32 & -6.66 & 1.12 & -3.10 & 0.32 & 5.1 & 173 & 3.23 \\
\hline \hline
\end{tabular}

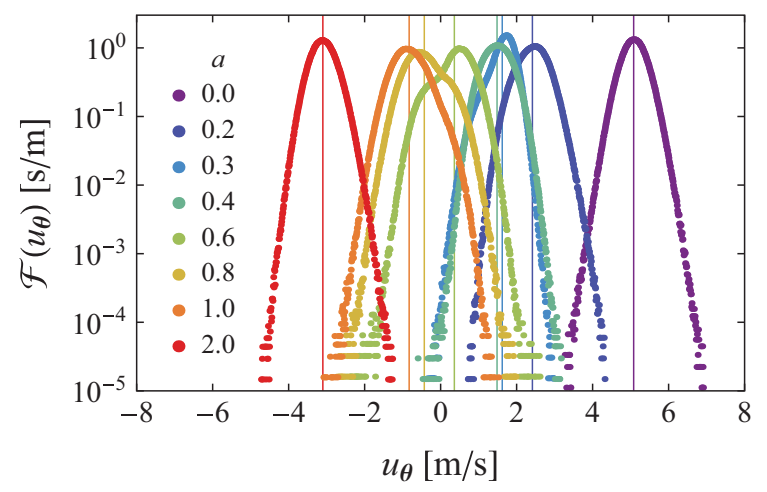

FIG. 2. (Color online) Probability density functions of the azimuthal velocity $\left[\mathcal{F}\left(u_{\theta}\right)\right]$ measured at mid-height for varying $a=-\omega_{o} / \omega_{i}$. The means of the velocities are indicated by grid lines with their respective color. $a$ increases from right to left.

temporal spacing. We chose double the frequency to also capture fast fluctuations which are otherwise lost. It can happen that for a relatively long time there is no measurement, while for other moments a burst of measurements is taken. We take care to disable parts of the interpolated time series for which the temporal gap in the original data is too big $(3 / f$ is chosen; although the effect is hardly noticeable we wanted to account for the time that the LDA beams are blocked by vertical supporting rods), and for which interpolation is not justified. These disabled data points are not used in any of the calculations, except for the spectra.

We will first look at the probability density function (PDF) of the velocimetry data; see Fig. 2. For the cases $a=0.6, a=$ 0.8 , and $a=1$, we see that the PDF $\mathcal{F}$ seems to be comprised of two parts, as it has two bumps; one bump for $u_{\theta}>0$ and one for $u_{\theta}<0$. It seems that the presence of a neutral line $\left(u_{\theta}=0\right)$ alters the flow dynamics, as was also found in Ref. [18]. The outer region is stabilized by the outer cylinder, while the inner region is destabilized by the inner cylinder. Somewhere in between there must therefore be an interface where $u_{\theta}=0$. For $a \in\{0.6,0.8,1.0\}$ our measurement position is on the border of this interface [18]. Also indicated in Fig. 2 and in Table I is the mean velocity. As expected, the mean velocity decreases monotonically with increasing $a$, while the standard deviation is quite similar throughout, but slightly higher for the cases where the PDF is comprised of two contributions [18].

In order to compare the shapes of the PDFs we standardize the velocities (transforming the data set such that it has zero mean and unit variance):

$$
\begin{aligned}
\mu_{n} & =\left\langle\left(u_{\theta}-\left\langle u_{\theta}\right\rangle_{t}\right)^{n}\right\rangle_{t}, \\
\sigma & =\sqrt{\mu_{2}}, \\
\tilde{u_{\theta}} & =\left\langle u_{\theta}-\left\langle u_{\theta}\right\rangle_{t}\right\rangle_{t} / \sigma,
\end{aligned}
$$

where $\langle\cdot\rangle_{t}$ denotes averaging over time. In Fig. 3 we plot the PDFs of the standardized velocities. We now see that the tails of most of the distributions behave much like Gaussians. This is also reflected in the value for the kurtosis $\left(\mu_{4} / \sigma^{4}\right)$; see Table I. The values that we find are close to 3 (except for the case $a=0.3$ ) and the distributions are only slightly leptokurtic or platykurtic. 


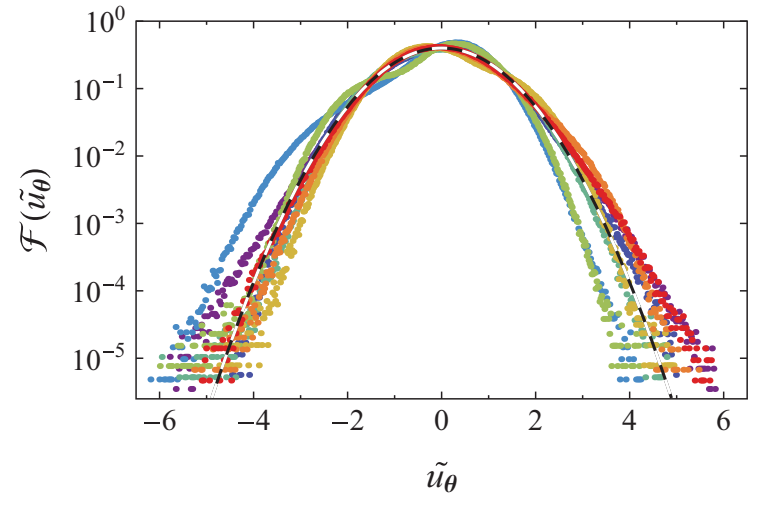

FIG. 3. (Color online) Probability density function of the standardized azimuthal velocity $\left[\mathcal{F}\left(\tilde{u_{\theta}}\right)\right]$ for various $a$. Colors are the same as in Fig. 2. The dashed line is a Gaussian with zero mean and unit variance.

Table I also includes the Taylor-Reynolds number $\operatorname{Re}_{\lambda}$. We find that the Taylor-Reynolds number is not necessarily the largest for the case when the torque is the highest $(a \approx 0.33)$, as a higher torque means a higher $\sigma$ [Eq. (3)], but also a higher $\epsilon$ [Eq. (2)]. Furthermore, we note that $\sigma$ depends on the radial and axial position and that therefore the Taylor-Reynolds number is a function of position.

Although the PDF of the velocity is of importance in describing a turbulent flow, it obviously does not describe the dynamics of the flow. We therefore look at velocity increments $\Delta_{r} u_{\theta}$ :

$$
\Delta_{r} u_{\theta} \equiv u_{\theta}(x+r)-u_{\theta}(x) .
$$

Here $r$ is the distance between the two measurement positions $x$ and $x+r$. As we probe the velocity at only one position, we have to invoke Taylor's frozen-flow hypothesis [19] to obtain the velocity increments: $u_{\theta}(x+r, t)=u_{\theta}(x, t-r / U)$ where $U$ is a typical velocity scale. Here we chose the rms velocity of $u_{\theta}$, as it best describes the displacement of a fluid parcel for the cases where the velocity is in both directions ( $a=0.6, a=$ 0.8 , and $a=1.0$ ). For the cases where the velocity is mainly in one direction the rms velocity is very close to the absolute of the mean velocity. We plot the PDF of $\Delta_{r} u_{\theta}$ [Eq. (4)] for several different $r$ for the cases of $a=0$ and $a=1$; see Fig. 4.

For both $a$ we can clearly see that for small $r / \eta_{K}$ the distributions are very leptokurtic (the kurtosis $\mu_{4} / \sigma^{4}=3.3 \times 10^{3}$ for $r / \eta_{K}=36, a=0$, and $\mu_{4} / \sigma^{4}=2.4 \times 10^{3}$ for $r / \eta_{K}=37$, $a=1$ ). Here $\eta_{K}$ is calculated based on globally measured torque values at the corresponding $a$. At $a=0$ the PDF does not recover to a Gaussian [Fig. 4(a)] for very large $r$, whereas it does become normally distributed for other $a$; see, e.g., Fig. 4(b) for $a=1$. We speculate that, for large $r$ and due to the periodic nature of our geometry and the coherent structures in our flow (Taylor vortices), the flow can stay correlated for an unusually long time for certain $a$ and for certain positions in the flow. We will systematically characterize the $r$ dependence of different moments using longitudinal structure functions: $D_{p}(r)=\left\langle\left(\Delta_{r} u_{\theta}\right)^{p}\right\rangle$. For odd moments $p, D_{p}(r)$ is converging very slowly; we therefore use the absolute $[20,21]$ values of the velocity increments:

$$
D_{p}^{*}=\left\langle\left|\Delta_{r} u_{\theta}\right|^{p}\right\rangle
$$
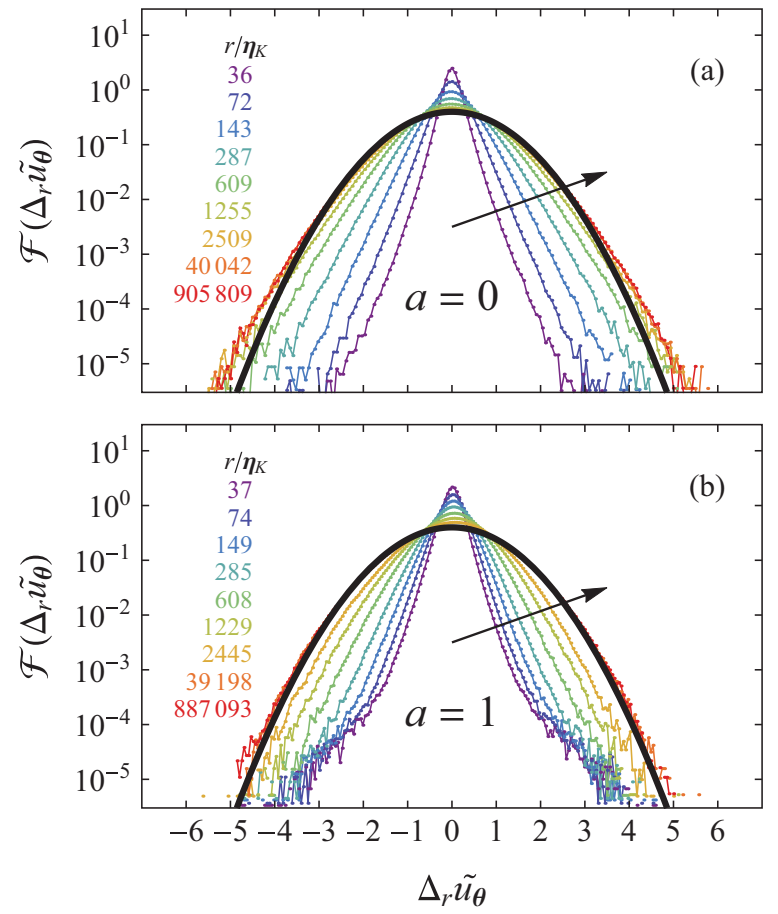

FIG. 4. (Color online) (a) Probability density functions of velocity increments $\left[\mathcal{F}\left(\Delta_{r} u_{\theta}\right)\right]$ for varying $r$ for the case of $a=0$. Values of $r$ are shown as multiples of the Kolmogorov length scale, and are colored accordingly. (b) As in (a) but for $a=1$. (a), (b) The black line is a Gaussian with zero mean and unit variance. The arrows indicate increasing $r / \eta_{K}$.

for all $p$. Note that there is no theoretical justification that $D_{p}^{*}(r)$ and $D_{p}(r)$ scale in the same way, although the scaling of $D_{p}^{*}$ (for odd $p$ ) has been found to be similar to that of $D_{p}$ but not essentially the same; see, e.g., [22,23]. Figure 5(f) shows the structure functions for $p \in[1,6]$ for the case of $a=0$. We carefully examine the convergence when computing the structure functions. While $D_{p}^{*}$ can be calculated using Eq. (5), we can alternatively express it as an integral:

$$
D_{p}^{*}=\int_{-\infty}^{\infty} \mathcal{F}\left(\Delta_{r} u_{\theta}\right)\left|\Delta_{r} u_{\theta}\right|^{p} d\left(\Delta_{r} u_{\theta}\right)
$$

We now take a careful look at the integrand of Eq. (6) and plot this integrand for points (a)-(e) in Fig. 5(f), corresponding to Figs. 5(a)-5(e), respectively. For increasing $p$ the structure functions measure the influence of increasingly rare events. We see in Figs. 5(a) and 5(b) that the tails of the integrands at points (a) and (b) are not fully converged, and we therefore have to omit these points from the structure functions. We do this analysis for all $p$ and for all $r$ for each case of $a$ and omit data from the analysis; these omitted data are plotted gray in Fig. 5(f). For high $p$ and low $r$ we find that we do not have sufficient statistics to capture all the rare events necessary to faithfully calculate $D_{p}^{*}$.

For fully developed turbulent flows the structure functions are suggested to scale as power laws in the inertial subrange [19]:

$$
D_{p}(r) \propto r^{\zeta_{p}}
$$



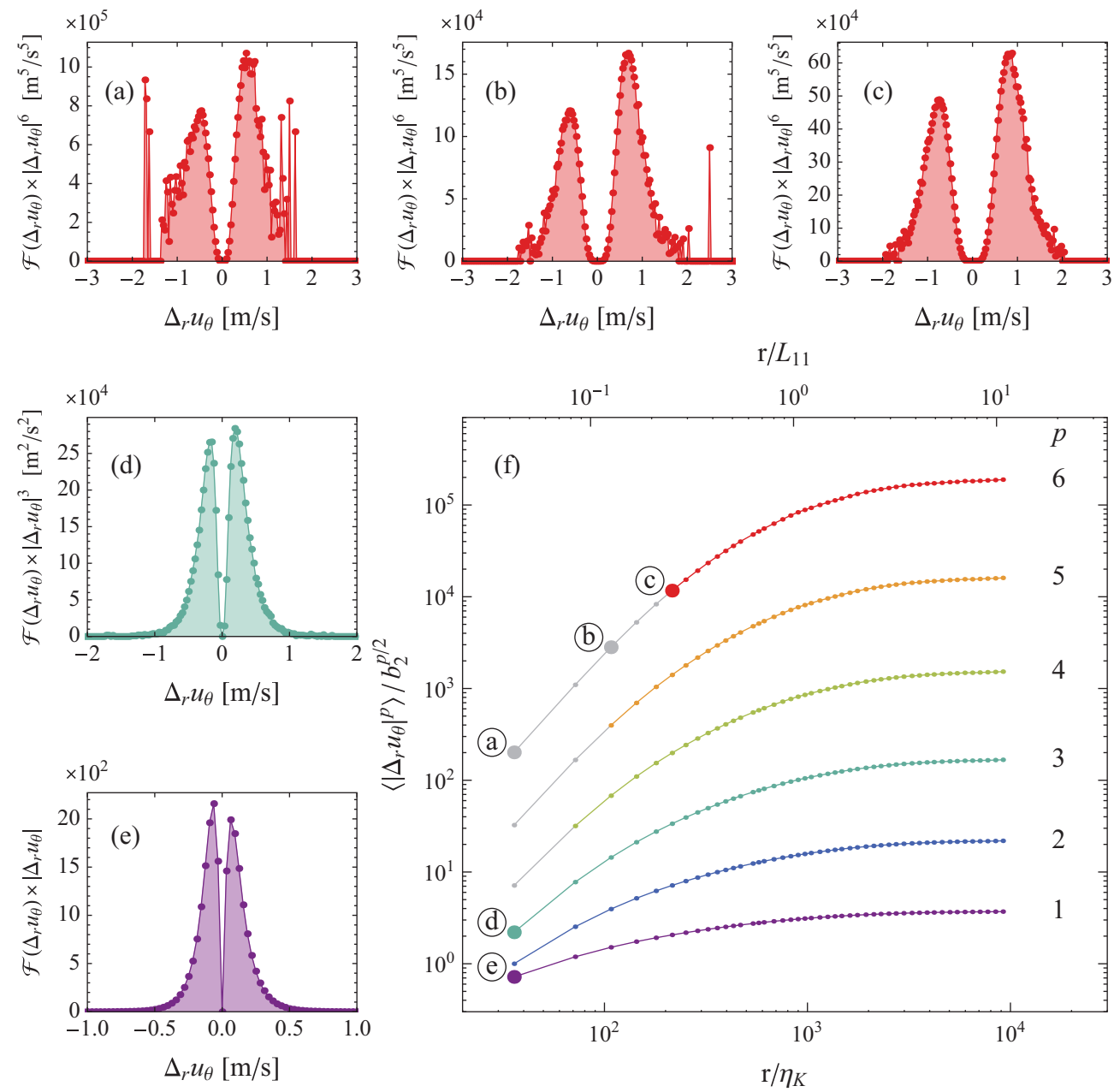

FIG. 5. (Color online) (a)-(e) Integrand of Eq. (6) for various positions marked in (f). The areas under the graphs are the values of $D_{p}^{*}(r)$. (a),(b) Nonconverged structure function integrands. (c)-(e) Converged structure function integrands. (f) Longitudinal structure functions ( $\left[D_{p}^{*}(r)\right]$ for varying powers $p$ (indicated on the right) for the case of $a=0$. Structure functions are scaled using a constant $b_{2}=D_{2}^{*}\left(r_{\text {min }}\right)$. The separation distance $r$ is normalized using the Kolmogorov length scale $\eta_{K}$ and the integral length scale $L_{11}$. For various $p$ and $r$ (thick points labeled (a)-(e) the integrand of Eq. (6) is plotted. Data in gray color are not fully converged, and are omitted in the ESS calculation.

Kolmogorov predicted [12,13,19] these exponents to scale as $\zeta_{p}=p / 3$; any deviation from this model is the result of the intermittency of the flow. For our case we will denote the scaling exponents as $\zeta_{p}^{*}$, as we are using $D_{p}^{*}(r)$ in the analysis rather than $D_{p}(r)$. We expect an inertial subrange for roughly $10 \eta<r<L_{11}$-where $L_{11}$ is the integral length

TABLE II. Structure function exponents $\zeta_{p}^{*}$, for different $a .0_{\mathrm{LS}}$ are the data from Lewis and Swinney [3], for which $a=0$ and $\operatorname{Re}=$ $5.4 \times 10^{5}$. Because of the usage of ESS by definition $\zeta_{3}^{*} \equiv 1$.

\begin{tabular}{ccccccccccc}
\hline \hline$a$ & 0 & $0_{\mathrm{LS}}$ & 0.2 & 0.3 & 0.4 & 0.6 & 0.8 & 1 & 2 \\
\hline$p$ & & & & & & & & & \\
1 & 0.37 & 0.37 & 0.37 & 0.37 & 0.39 & 0.37 & 0.37 & 0.37 & 0.37 \\
2 & 0.70 & 0.70 & 0.71 & 0.71 & 0.72 & 0.70 & 0.71 & 0.70 & 0.70 \\
$3^{*}$ & 1 & 1 & 1 & 1 & 1 & 1 & 1 & 1 & 1 \\
4 & 1.27 & 1.27 & 1.27 & 1.26 & 1.25 & 1.26 & 1.26 & 1.27 & 1.28 \\
5 & 1.51 & 1.50 & 1.51 & 1.50 & 1.49 & 1.53 & 1.51 & 1.53 & 1.53 \\
6 & 1.71 & 1.72 & 1.73 & 1.71 & 1.70 & 1.69 & 1.71 & 1.78 & 1.77 \\
\hline \hline
\end{tabular}

scale-because these length scales are separated by roughly two decades for our flow. But, as seen in Fig. 5(f), we do not see an inertial subrange where the structure functions show power-law scaling, a finding also observed in Ref. [3]. We therefore are unable to extract structure function exponents directly from our structure functions; this results holds for all our $a$. As suggested by Benzi et al. [15], we can extend the range over which scaling holds by employing extended self-similarity (ESS). The $p$ th-order structure function is plotted as a function of the third-order structure function; the scaling exponent is now given by $\zeta_{p}^{*} / \zeta_{3}^{*}$. From the NavierStokes equations one can derive the Kolmogorov-Howardvon Kármán $(\mathrm{KHvK})$ relation, from which we can determine that for $r \gg v^{3 / 4} \epsilon^{-1 / 4}, D_{3} \propto-\frac{4}{5} \epsilon r$, or $\zeta_{3}=1$. In this work we will assume that $\zeta_{3}=\zeta_{3}^{*}=1$, and we would like to note that the $\mathrm{KHvK}$ relation is derived under the assumption of isotropic homogeneous turbulence, which is questionable in our flow arrangement. Nevertheless, we employ ESS analysis for our longitudinal structure functions; see Figs. 6(a) $(a=0)$ and 7(a) $(a=1)$. 

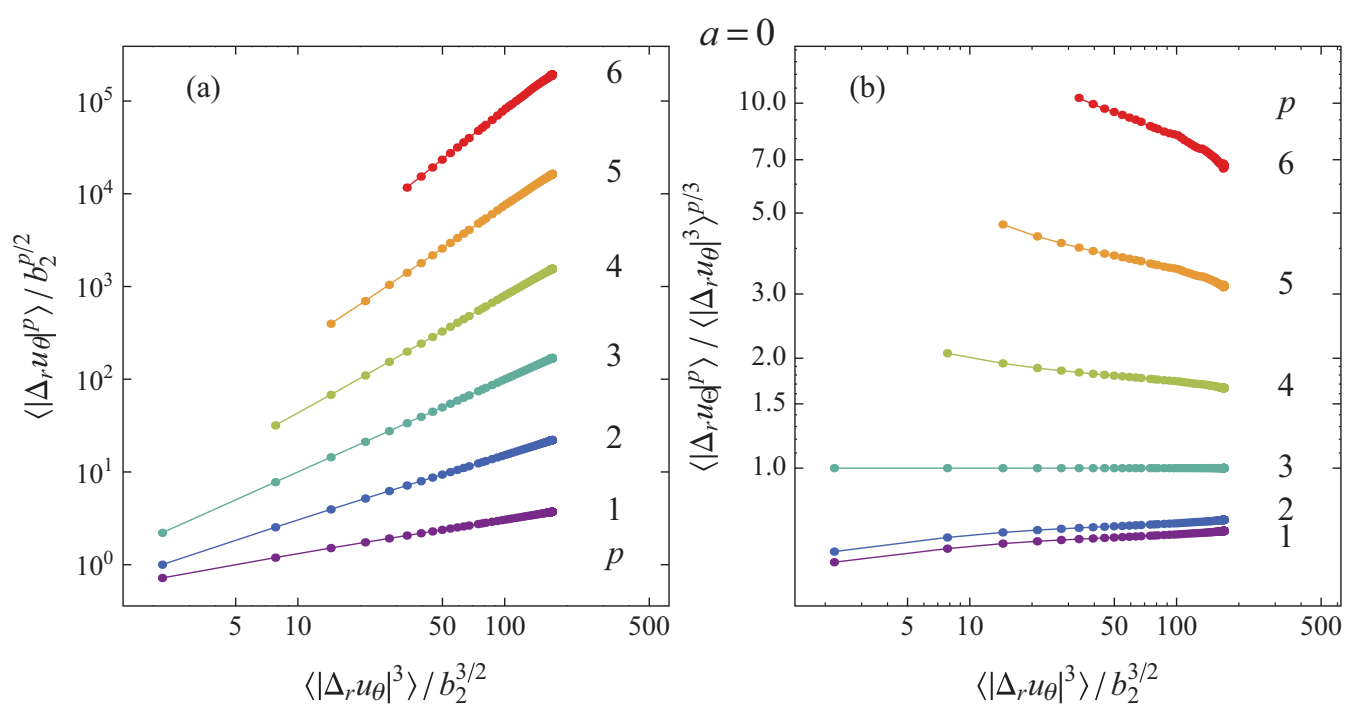

FIG. 6. (Color online) (a) Extended self-similarity for the case of $a=0$. Powers $p$ are indicated on the right. Power-law scaling can be seen for each power $p$. (b) Extended self-similarity compensated by Kolmogorov scaling $p / 3$. The quality of the scaling can now be seen better. Gray data in Fig. 5(f) are omitted in this analysis as they are not fully statistically converged.

We now clearly see that in the ESS representation the scaling is much better, and that we are able to extract structure function exponents from our data. In addition, we also compensate our data with Kolmogorov's prediction $(p / 3)$; see Figs. 6(b) and 7(b). Note that in these compensated plots $[20,21]$ (plotted on double-logarithmic scales) any deviation from a perfect power scaling is amplified and clearly visible. Furthermore, we expect a straight line in the case of perfect scaling. We perform power-law fits to our ESS data for all $a$, and extract $\zeta_{p}^{*}$; see Table II and Fig. 8.

Lewis and Swinney [3] have performed a similar analysis on Taylor-Couette flow for pure inner cylinder rotation, and we have included their structure function exponents (obtained using ESS, $\operatorname{Re}=5.4 \times 10^{5}$ ) also in the aforementioned table.
We see that for $1 \leqslant p \leqslant 5$ the structure function exponents are similar, independent of the Reynolds number, and almost independent of the amount of counter-rotation applied to the system. It is only for $p=6$ that the differences between the exponents become noticeable; 1.69 for $a=0.6$ and 1.78 for $a=0$. This difference might be caused by not including the high- $p$ and low- $r$ data in our fits due to lack of statistics. We notice that for increased counter-rotation (i.e., increased $a$ ) we do not see a systematic trend for the structure function exponents. From global torque measurements $[6,7,18,24]$ we know that there is a maximum in the torque needed to sustain a constant angular velocity for both cylinders. This peak in the torque has been found around $a \approx 0.33$. This peak in the torque has as a consequence that the Kolmogorov length scale is smallest for
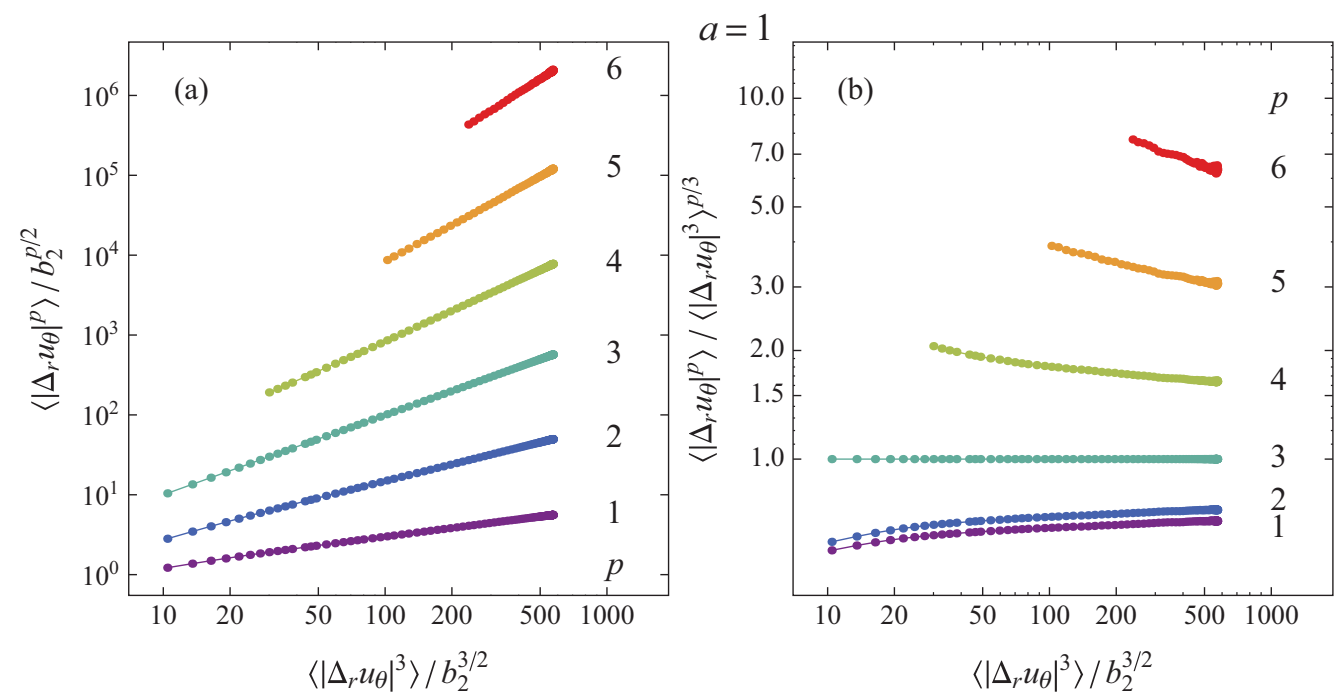

FIG. 7. (Color online) (a) Extended self-similarity for the case of $a=1$. Powers $p$ are indicated on the right. Power-law scaling can be seen for each power $p$. (b) Extended self-similarity compensated by Kolmogorov scaling $p / 3$. The quality of the scaling can now be seen better. As in the analysis of Fig. $5(a=0)$, nonconverged data are omitted from the structure functions. 


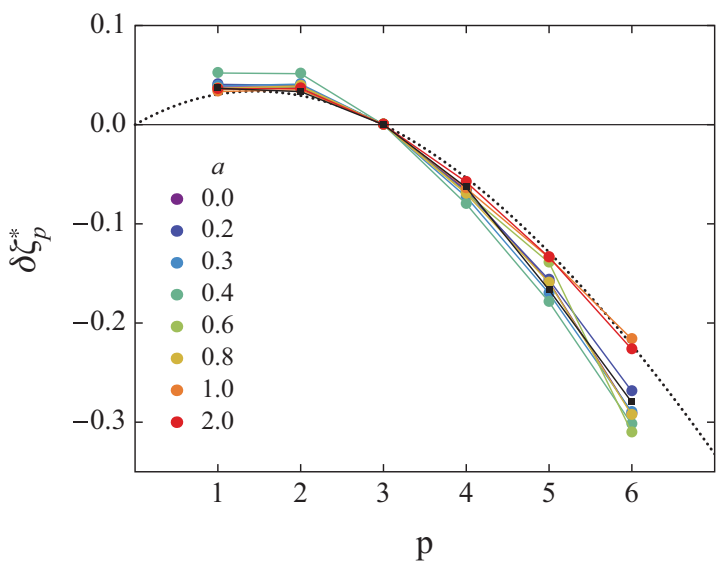

FIG. 8. (Color online) Longitudinal structure function exponents deviations: $\delta \zeta_{p}^{*}=\zeta_{p}^{*}-p / 3$. Black dotted line shows the SheLévêque model $\left[\zeta_{p}=p / 9+2+2(2 / 3)^{p / 3}\right]$. Black squares are the data from Lewis and Swinney [3] for $a=0$.

the rotation ratio $a=0.3\left(\eta_{K}=15.7 \mu \mathrm{m}\right)$ and largest for $a=$ $2.0\left(\eta_{K}=21.0 \mu \mathrm{m}\right)$ - assuming homogeneous turbulence for both cases. We, however, do not see any similar trend in the structure function exponents. It seems that all the exponents are nearly universal; independent of the Reynolds number (Lewis and Swinney [3] also performed their experiments for $\mathrm{Re}=$ $6.9 \times 10^{4}$ and found similar structure function exponents as for our $\operatorname{Re}=1.38 \times 10^{6}$ ) and independent of the amount of counter-rotation applied to the system. Our structure functions are tabulated in Table II and plotted in Fig. 8; for comparison we have included the data from Lewis and Swinney [3] and the She-Lévêque model with its standard parameters [25]. We find that, for nearly all the exponents and for nearly all the $a$ cases, Taylor-Couette flow seems more intermittent than the She-Lévêque model predicts, as already reported in Ref. [3].

In Fig. 9(a) we plot the spectra for all cases. We see that most of the energy is kept in the low frequencies [26], even lower than the driving frequency: $\omega_{i}-\omega_{o}=2 \pi \times 10 \mathrm{~s}^{-1}$ for the cases $a \in\{0.6,0.8,1.0\}$. Furthermore, we see for $a=0$ that we do not have any power-law scaling behavior, as was already found in the TC experiments of Ref. [3]. However, for $a \in\{0.6,0.8,1.0\}$ - the same $a$ 's for which the PDF of the azimuthal velocity showed that it was made up of two distributions-we see a power-law scaling with exponent $-5 / 3$. To reveal the quality of the scaling we compensate the data by $\omega^{5 / 3}$; see Fig. 9(b). We indeed see in Fig. 9(b) that the power spectra for $a \in\{0.6,0.8,1.0\}$ level out over roughly two decades. Furthermore, for the case $a=0$ we see that there is no scaling whatsoever. In addition, we also plot the local slope of the spectrum. Here we also see that the power-law scaling for $a \in\{0.6,0.8,1.0\}$ is around $-5 / 3$ close to $\omega=10^{3} \mathrm{~s}^{-1}$, and that for $a=0$ the exponent is constantly changing. The curving up of our spectra at the high-frequency end is due to our limited measuring frequency. We therefore do not recover the steep slopes $(\gg 2)$ found by Lewis and Swinney [3]. However, they also found that for $a=0$ the local slope is never constant and is a monotonic function of $\omega$.

In this work we relied on Taylor's hypothesis, which certainly has to be experimentally justified in the future using a field measurement technique, e.g., particle imaging velocimetry. In addition we assumed concepts of homogeneous isotropic turbulence to obtain $\epsilon$ and therefore $\eta$. Future work will be necessary to study the anisotropic properties of the flow $[27,28]$.

To summarize, we have measured the local azimuthal velocity in a turbulent Taylor-Couette flow with various amounts of counter-rotation using laser Doppler anemometry. We found that the structure functions show no inertial subrange for all $p$ and for all cases $a$. Using extended self-similarity analysis [15], we extracted the structure function exponents, which are in good agreement with earlier results by Lewis and Swinney [3]. We find that for $a=0$ the structure function exponents are nearly independent of the Reynolds number: previous results [3] are for $\operatorname{Re}=6.9 \times 10^{4}$ and $\operatorname{Re}=5.4 \times 10^{5}$, while our current results are for $\operatorname{Re}=1.38 \times 10^{6}$. Any discrepancy between these exponents could easily be caused by different
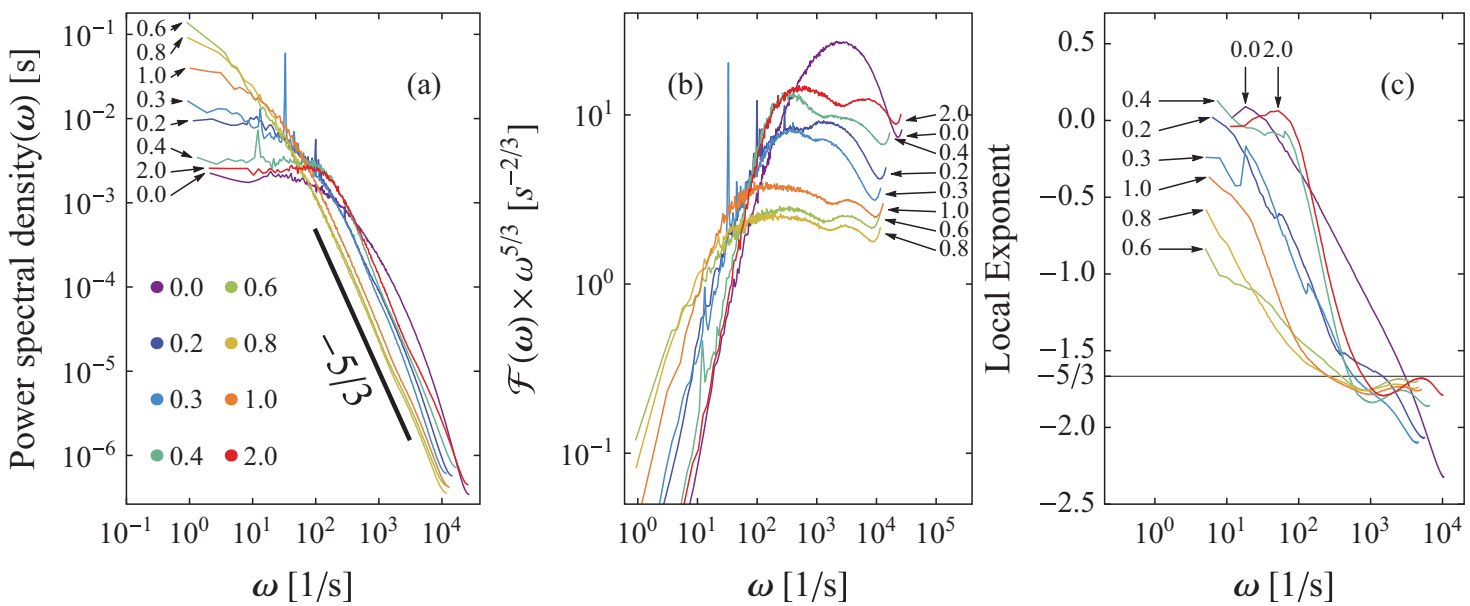

FIG. 9. (Color online) (a) Spectral density of the azimuthal velocity for various $a ;-5 / 3$ scaling is indicated with a black line. (b) Spectral density compensated by $\omega^{5 / 3}$. (c) Local scaling exponent over an interval of one decade as a function of frequency $\omega$. Kolmogorov's inertial subrange scaling $(-5 / 3)$ is included as a horizontal line. The legend displayed in (a) corresponds also to the colors used in (b) and (c). Arrows indicate the corresponding $a$. 
fitting intervals and are certainly within experimental error. Furthermore, we find that adding rotation of the outer cylinder of the system to create counter-rotation does not strongly influence the structure function exponents, but does strongly change the scaling of the spectra. While for $a \in\{0.6,0.8,1.0\}$ we see a clear power-law scaling in the spectra, we do not observe such clear scaling in the second-order structure function.

\section{ACKNOWLEDGMENTS}

We acknowledge the assistance of B. Basel and $\mathrm{H}$. Ligtenberg, and we thank B. Benschop, M. Bos, and G. W. Bruggert for their technical support. This study was financially supported by the Simon Stevin Prize of the Technology Foundation STW of The Netherlands.
[1] G. I. Taylor, Philos. Trans. R. Soc., A 223, 289 (1923).

[2] D. P. M. van Gils, G. W. Bruggert, D. P. Lathrop, C. Sun, and D. Lohse, Rev. Sci. Instrum. 82, 025105 (2011).

[3] G. S. Lewis and H. L. Swinney, Phys. Rev. E 59, 5457 (1999).

[4] B. Dubrulle, O. Dauchot, F. Daviaud, P.-Y. Longaretti, D. Richard, and J.-P. Zahn, Phys. Fluids 17, 095103 (2005).

[5] H. Ji, M. Burin, E. Schartman, and J. Goodman, Nature (London) 444, 343 (2006).

[6] D. P. M. van Gils, S. G. Huisman, G. W. Bruggert, C. Sun, and D. Lohse, Phys. Rev. Lett. 106, 024502 (2011).

[7] M. S. Paoletti and D. P. Lathrop, Phys. Rev. Lett. 106, 024501 (2011).

[8] S. G. Huisman, D. P. M. van Gils, S. Grossmann, C. Sun, and D. Lohse, Phys. Rev. Lett. 108, 024501 (2012).

[9] S. Merbold, H. J. Brauckmann, and C. Egbers, Phys. Rev. E 87, 023014 (2013).

[10] H. J. Brauckmann and B. Eckhardt, J. Fluid Mech. 718, 398 (2013).

[11] B. Eckhardt, S. Grossmann, and D. Lohse, J. Fluid Mech. 581, 221 (2007)

[12] A. N. Kolmogorov, Dokl. Akad. Nauk. SSSR 30, 299 (1941).

[13] A. N. Kolmogorov, Dokl. Akad. Nauk. SSSR 31, 538 (1941).

[14] Z.-S. She, K. Ren, G. S. Lewis, and H. L. Swinney, Phys. Rev. E 64, 016308 (2001).
[15] R. Benzi, S. Ciliberto, R. Tripiccione, C. Baudet, F. Massaioli, and S. Succi, Phys. Rev. E 48, R29 (1993).

[16] Dantec, PSP-5, polyamide seeding particles, $2.5 \mu \mathrm{m}$ diameter.

[17] S. G. Huisman, D. P. van Gils, and C. Sun, Eur. J. Mech. B/Fluids 36, 115 (2012).

[18] D. P. M. van Gils, S. G. Huisman, S. Grossmann, C. Sun, and D. Lohse, J. Fluid Mech. 706, 118 (2012).

[19] U. Frisch, Turbulence (Cambridge University Press, Cambridge, 1995).

[20] S. Grossmann, D. Lohse, and A. Reeh, Phys. Fluids 9, 3817 (1997).

[21] S. Grossmann, D. Lohse, and A. Reeh, Phys. Rev. E 56, 5473 (1997).

[22] G. Stolovitzky and K. R. Sreenivasan, Phys. Rev. E 48, R33 (1993).

[23] J. Herweijer and W. van de Water, Phys. Rev. Lett. 74, 4651 (1995).

[24] R. Ostilla, R. J. A. M. Stevens, S. Grossmann, R. Verzicco, and D. Lohse, J. Fluid Mech. 719, 14 (2013).

[25] Z.-S. She and E. Leveque, Phys. Rev. Lett. 72, 336 (1994).

[26] M. López-Caballero and J. Burguete, Phys. Rev. Lett. 110, 124501 (2013).

[27] S. Grossmann, D. Lohse, and A. Reeh, J. Stat. Phys. 93, 715 (1998).

[28] L. Biferale and I. Procaccia, Phys. Rep. 414, 43 (2005). 